\title{
THE CONSTRUCTION OF 4 DENSE ASTROMETRIC STANDARD AREAS WITH THE CARLSBERG AUTOMATIC MERIDIAN CIRCLE
}

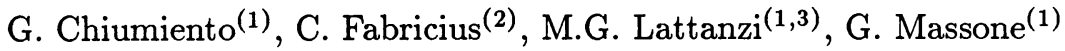 \\ (1) Torino Astronomical Observatory, 10025 Pino Torinese (TO), Italy \\ (2) Copenhagen University Observatory, Brorfelde, Denmark \\ (3) Space Telescope Science Institute, Baltimore MD 21218, USA
}

High precision work in photographic astrometry requires accurate monitoring of the telescope+filter+emulsion system being used.

Usually, this is done via calibration stars in standard regions, i.e., by assuming the geometry of the field as perfectly known during the reduction of plates specially taken for this purpose. Moreover, such areas can serve as testing regions for plate modelling improvements. The available material is either quite old or only covers small areas of the sky.

The high accuracy required in linking the Extragalactic Reference Frame and the Optical Reference Frame, the recent acquisition of fully digitized deep Schmidt sky surveys and the HIPPARCOS mission are demanding a decisive improvement of the present situation.

Our project is the realization of 4 astrometric regions by using the Carlsberg Automatic Meridian Circle (CAMC) located at La Palma, Canary Islands (Spain). The selected fields and their main characteristics are given in the table below. These areas are located near the equator and uniformly distributed in R.A. The star density varies across the areas (denser toward the centre) because we also require different concentric fields inside the areas (i.e., the fields of long-focus and wide-field telescopes) to be uniformly covered with adequate density. Approximate positions and magnitudes (close to the $\mathrm{V}$ band) are from the Guide Star Catalog (Space Telescope Science Institute, 1989). The magnitude range of the selected stars is between 10.0 and $14.5^{m}$.

Proper motions will be determined for stars found in the Astrographic Catalogue. Precise determination of $\mathrm{B}$ and $\mathrm{V}$ magnitudes is also planned.

$\begin{array}{cccc}\text { STANDARD } & \text { FIELD CENTRE } & \text { SIZE } & \text { NUMBER } \\ \text { AREAS } & \text { R.A. (J1989.5) DEC. } & \text { (DEG) } & \text { OF STARS }\end{array}$

$\begin{array}{lrrrrrr}\text { QSO 0111+021 } & 1^{h} & 13.2^{m} & 2^{\circ} & 18^{\prime} & 7 \times 7 & 316 \\ \text { NGC 2244 } & 6 & 31.8 & 4^{\circ} & 52^{\prime} & 10 \times 8 & 388 \\ \text { QSO 1148-001 } & 11 & 50.2 & -0^{\circ} & 20^{\prime} & 7 \times 7 & \approx 310 \\ \text { IC } 4756 & 18 & 38.4 & 5^{\circ} & 26^{\prime} & 8 \times 8 & 324\end{array}$

